\title{
Medicina y literatura en la formación del médico residente de medicina interna
}

\author{
F. J. BARBADO HERNÁNDEZ
}

Servicio de Medicina Interna. Hospital Universitario La Paz. Universidad Autónoma de Madrid

\begin{abstract}
RESUMEN
Las humanidades médicas son inseparables de la actividad clínica. El objetivo de este trabajo es demostrar que la medicina basada en pruebas y la medicina humanitaria basada en narrativas y valores deben ir necesaria y eficazmente juntas. Se detalla para qué sirve la literatura en la formación del médico residente de medicina interna y se expone como metódica un programa de docencia complementaria sobre medicina y literatura. Las obras literarias pueden hacer al lector médico, un "mejor médico".
\end{abstract}

PALABRAS CLAVE: Literatura. Medicina. Médico residente. Formación.
MEDICINE AND LITERATURE IN THE TRAINING OF A HOUSE PHYSICIAN AN INTERNAL MEDICINE

\begin{abstract}
Medical humanities are inseparable from the clinical activity. The aim of this paper is to demonstrate that evidence-based medicine and humanitary medicine based on narratives and values must be necessarily en effectively joined. It is detailed the utility of literature in the training of a house physician in internal medicine and it is exposed as methodology a complementary learning program about medicine and literature. Literary works are able to do from a reader physician, a "better physician".
\end{abstract}

KEY WORDS: Literature. Medicine. House physician. Training.

Barbado Hernández. FJ. Medicina y literatura en la formación del médico residente de medicina interna. An Med Interna (Madrid) 2007; 24: 195-200.

\section{INTRODUCCIÓN}

Cuando los coordinadores de las II Jornadas de Actualización en Enfermedades Infecciosas, organizadas por los Médicos Residentes (MIR) de Medicina Interna del Hospital "La Paz", Mayo-2006, me propusieron como tema de la Sesión Inaugural: "El Arte en las Enfermedades Infecciosas", me sorprendió. Pero enseguida me dejaron elegir lo que más me gusta: la clínica, "ver enfermos", la realización de historias clínicas, la formación del MIR y de los alumnos y ¡relacionarlo con la literatura!

¿Heteróclito título de esta ponencia? Ustedes están pensando ¿literatura en tiempos de la Medicina Basada en la Evidencia, de la Red de Redes, del Estatuto -idel MIR!-, de las "lágrimas perversas" descritas en las Cartas al Director de un diario de tirada nacional? (1). La propuesta de la Sesión me ha dejado "el desasosiego de Pessoa" (2). En el final del otoño de la vida (3) ¿me habrán enviado al Arte y la Literatura? El tiempo ha llegado. Pero, parodiando a Neruda (4), confieso que he vivido como bibliófilo, pero no bibliómano, impenitente buscador de libros -es otra cosa: coleccionista per se (5)-, asiduo de librerías de lance, rastrillos, paseante con joroba barojiana de la Cuesta Moyano (6). Por cierto que en estas caminatas he advertido que lo primero que hacen algunas viudas de médicos es vender su biblioteca personal.

Voy a intentar persuadirles. Las Actividades Complementarias Comunes a la Formación Específica del MIR, entre las que destacan las Humanidades Médicas, son inseparables de la actividad clínica. Mi objetivo es demostrar que las dos tendencias actuales, la medicina basada en pruebas, por un lado, y la medicina humanística basada en narrativas y valores, por otro, deben ir necesaria y eficazmente juntas $(7,8)$. 


\section{¿CUÁL ES EL ORIGEN DE LA INQUIETUD LITERARIA DEL MÉDICO?}

Ustedes ya saben lo que es la Medicina, lo han leído en el Harrison al preparar el examen MIR: "una combinación de conocimientos científicos, habilidades técnicas, arte clínico y actitudes humanitarias" (9). Y el arte de la medicina - itodavía existe!- es "la combinación de conocimientos médicos, intuición, experiencia y buen juicio" y tiene la misma trascendencia que la medicina como ciencia de firmes fundamentos (9). En esta mezcla de ciencia, cultura y humanidades ¿por qué la fascinante sugestión que la literatura ejerce sobre los médicos? Existe una respuesta universal: por el contacto diario con el enfermo, con sus miserias, sus inquietudes, angustias y temores acerca de la enfermedad y de la muerte $(8,10-12)$. El catálogo de médicos-escritores y escritores-médicos es extenso $(13,14)$. La síntesis es Anton Chejov (1860-1904) que consideró a la Medicina como la esposa legítima y a la Literatura la amante, con fraseología de otros tiempos $(15,16)$.

\section{EL MÉDICO, HUMANISMO Y HUMANIDADES MÉDICAS}

Existen excelentes trabajos y monografías sobre estas cuestiones $(8,11,13,17-21)$. Como médico de enfermos, sólo voy a mencionar una cita de Lorenzo-Cáceres (17) sobre un médico de familia, protagonista de la novela La enfermedad de Sachs, de Martin Winckler (23). Dice el Dr. Sachs: "pero en todos estos años jamás me han dicho que podía sentarme a la cabecera de un moribundo y sujetarle la mano y hablarle." Y además, esto nos parece propio de familiares y enfermeras.

¿REALMENTE SIRVE PARA ALGO LA LITERATURA EN LA FORMACIÓN DEL MÉDICO RESIDENTE DE MEDICINA INTERNA?

Es decir ¿por qué y para qué?. La literatura constituye un recurso docente eficaz para algunos aspectos ignorados en las Facultades $(10,17)$. Fernando Navarro escribía recientemente (24): "No es nada raro que en el ejercicio de su profesión, el médico busque los aspectos más profundos de la enfermedad, el dolor, el sufrimiento, la locura y la muerte, los sentimientos o sensaciones más íntimos. En esta búsqueda, los libros de texto son de escasa utilidad: es necesario acudir a la literatura universal." Y escoge como excelente ejemplo La peste de Albert Camus (25).

Para Castilla del Pino (26): "la literatura es una forma de conocimiento de la vida superior a la de la vida propia. Conocemos más de los personajes literarios que de aquellos con los que vivimos." Según este médico-psiquiatra podríamos llegar a conocer a Madame Bovary mejor que a nuestra propia mujer.

William Osler en su libro Aequanimitas (27) sostiene la teleología de este trabajo: "para comprender los temores, las preocupaciones y los conflictos emocionales de los enfermos, a veces no hay mejor fuente de información que la literatura."

\section{¿PARA QUÉ LITERATURA Y MEDICINA?}

1. Como complemento para adquirir las habilidades clínicas generales, entre las que destaca la capacidad para obtener una historia clínica con todos los detalles relevantes (28). Dice Laín (20): "una historia clínica qué es si no el relato de los sucesivos encuentros entre dos personas, el médico y el enfermo." Es fascinante la interpretación literaria que hace el escritor J.J.Millás (29) de la historia clínica: "he leído muchos historiales de médicos y puedo asegurar que los mejores, incluso desde el punto de vista de la clínica, eran verdaderas novelas, mientras que las verdaderas novelas siempre tienen, más o menos oculta, una vocación de historia clínica."

Quiero resaltar al MIR de Medicina Interna que además de saber qué enfermedad tiene el enfermo, es necesario conocer quién tiene la enfermedad. Algo tan inmutable como la Medicina de la biografía y de la unidad psicofísica del enfermo, es decir la patobiografía, es bien conocido en la literatura (30-33).

2. Para adquirir habilidades comunicativas (28), capacidad para detectar las principales preocupaciones y expectativas de los enfermos y transmitir esperanza y compasión. El médico "es un posibilitador de esperanzas." (34). ¡Cuidado! Las palabras del médico pueden ser "oro molido" o ser "palabras como bisturíes" (35). Axel Munthe en su inquietante autobiografía La historia de San Michele (36) nos muestra que "no hay ninguna cosa tan poderosa como la esperanza."

3. La literatura nos ayuda a conocer las enfermedades desde "otras miradas" (37).

Por ejemplo, para internistas e infectólogos, la tuberculosis. Si nos adentramos en la densa Montaña Mágica (1924), o no hemos llegado al final, del alemán Tomás Mann (38), en mi opinión la mejor descripción del enfermo con tuberculosis (psicología, sentimientos e incluso semiología clínico-literaria) es de Cela en Pabellón de reposo (1943) (39).

Estoy de acuerdo con Luis Landero (40) cuando considera que el mejor libro sobre la enfermedad es La muerte de Iván Ilich de León Tolstoi (41). Es un relato -iuna auténtica patobiografía!- insuperable, conmovedor y sobrecogedor sobre la psicología del enfermo con una enfermedad mortal. Es un relato excelente para la propuesta de Terés et al. (28), sobre la adquisión de habilidades y actitudes para la atención al paciente terminal.

Un detalle: existen enfermedades "nacidas o descritas" en la literatura, por ejemplo el síndrome de Picwick en los Papeles Póstumos del Club Picwick, de Dickens (42), el síndrome de Stendhal (43), o la enfermedad de Tomás (44).

4. Literatura y drogas.

Basta mencionar al penetrante novelista Aldous Huxley, quien en dos ensayos, Las puertas de la percepción y Cielo e infierno, analiza de forma magistral los efectos subjetivos de las drogas (45). Una familia que debe conocer el Médico Residente: Aldous Huxley fue nieto del médico y naturalista Thomas Henry Huxley (1825-1895), coetáneo y defensor de las teorías de Darwin, hermano del biólogo Julián Huxley y hermanastro del Premio Nobel de Fisiología Andrew Huxley. Esta familia es símbolo y síntesis de ciencia, medicina y literatura (46-48)

5. Las autobiografías y las memorias, sobre todo de médicos, son de gran interés y enseñanza. Su lectura significa conocer y vivir otras experiencias que enriquecen la nuestra.

Axel Munthe, médico psiquiatra sueco, en la mencionada Historia de San Michele (36) realiza un apasionante recorrido por la Medicina y los enfermos del siglo XIX, una perspectiva 
distinta a la histórica $(49,50)$ de Charcot y de sus discípulos, Freud y Babinski, en la Salpetrière de París, ya entonces ¡la ciudad más hermosa del mundo!

Castilla del Pino en sus brillantes Memorias Pretérito imperfecto (51) lleva la sinceridad y veracidad hasta el desgarro, quizás sea la única forma de conocer "por dentro" la Medicina española de la primera mitad del siglo XX, y poder contrastar con otras visiones contrapuestas (34).

Don Pío Baroja en sus memorias Desde la última vuelta del camino (6) y sobre todo en El árbol de la ciencia (52), novela en buena medida autobiográfica y tristemente obligatoria en el Bachillerato, proyecta de forma permanente su ego de médico. En su novela El mundo es ansí (53) refleja de forma exacta cómo la vida es sufrimiento y dolor: "dureza, crueldad, egoísmo e ingratitud." Quizás por ello las obras de Baroja, desde hace más de 100 años, conservan frescura y sorprendente polémica permanente (54).

Un escritor, no médico, Stendhal, pseudónimo de Henry Boyle, definió a la novela como "un espejo que se pasea por el camino" Tiene extraordinario interés para el médico en formación su "ver en lo que es", con sus penetrantes y complejos análisis de la realidad en su autobiografía Vida de Henri Brulard (55).

En mi opinión, la mejor Medicina desnuda, a campo abierto, desprovista de hospitales y tecnologías, está en dos libros cautivadores: Morfina, del médico ruso Bulgakov (56) y Escenas de la vida de un médico, del médico portugués Fernando Namora (57). (28)

6. Capacidad para el análisis crítico durante la formación

Afirma Rozman que "uno de los principios esenciales de la práctica clínica estriba en dudar de todo, es decir tener consciencia sobre la gran dosis de incertidumbre que suele asociarse al ejercicio clínico" (58), actitud que la literatura, como la experiencia, nos puede enseñar. El MIR de Medicina Interna es un futuro especialista en incertidumbres.

Para Jorge Wagensberg (59): "el especialista menos regresivo es el especialista en incertidumbre, es decir el generalista."

\section{LOS OTROS: CIENTIIFICOS QUE RECHAZAN LA LITERATURA}

Es frecuente la expresión, entre científicos básicos, "leer una novela, por ejemplo, es perder el tiempo."

La bióloga Lynn Margulis, pionera de la simbiogénesis, en una reciente Conferencia en Madrid (60) afirmó: "la única noticia es la ciencia, lo demás es chismorreo." Eudald Carbonell, en su extraordinario libro Los sueños de la evolución (61) escribe: "a pesar de que no tengo costumbre de leer libros de ficción y que conste que lo he intentado." Sin embargo, este autor es fascinante en sus libros de divulgación científica (Las claves del pasado, La llave del futuro, Sapiens, Planeta humano).

Es interesante la reflexión del físico e historiador Sánchez Ron (62): "entre muchos científicos de nuestro siglo, ha proliferado una actitud, o filosofía, según la cual uno de los principales atractivos de la ciencia es el de que constituye una huida de la vida diaria con su dolorosa crudeza y desoladora vaciedad." Esta vaciedad también se puede atenuar con la irónica sentencia de Franzen (63): "es mejor refugiarse en la literatura que en la familia."
LA METÓDICA: EL CÓMO DE LA FORMACIÓN COMPLEMENTARIA EN MEDICINA A TRAVÉS DE LA LITERATURA

Rof Carballo (31) cita que cuando un estudiante pidió a Sydenham le recomendase el mejor libro para entrenarse en la práctica de la medicina, replicó Sydenham: "Leed el Quijote"

En la tabla I se muestra la propuesta de un esbozo de un Programa de Docencia Complementario sobre Literatura y Medicina.

1. Clases Prácticas a los alumnos de $3^{\circ}$ Curso en Patología General.

En grupos reducidos durante el aprendizaje de la historia clínica y Propedéutica. Es la mejor época, con la sensualidad involuntaria de sus gloriosos veinte años, para aventuras literarias en Medicina.

2. Pases de visita en la Sala.

¿Por qué se debe hablar, con humor y desenfado, en la visita hospitalaria, de la actualidad, y de literatura si las circunstancias se relacionan? Sencillamente porque mejora la relación médico-enfermo (64) y para enseñar habilidades comunicativas, como la capacidad para generar una mutua confianza con los enfermos y sus familiares (28).

¿Sirve para algo preguntar al MIR, a los alumnos, sobre áreas relacionables entre Medicina y Literatura? Sin duda, es un importante estímulo para su formación y expresa la fecunda simbiosis de literatura y práctica médica.

3. Sesiones clínicas paralelas.

Como ejemplo, podemos citar al profesor Letona, quien en una sesión sobre La enfermedad en la novela de Miguel Delibes (65) realiza un magnífico y didáctico diagnóstico clínico diferencial en torno a qué le pasa a Teodomira en El hereje: un discutible panhipopituitarismo o síndrome de Sheehan.

Prefiero estas Sesiones de Papel -o un Club del Papel barojiano $(54,66)$ - con enfermos de creación literaria a la enseñanza clínica con actores que representan a falsos enfermos (67). Si un día en mis andanzas de ponente en sesiones "ciegas" $(68,69)$, me encuentro con un actor como falso enfermo: no lo duden, le diagnosticaré de "enfermedad facticia."

4. Textos literarios.

Joseph E. Baños (10) realiza una excelente propuesta de un Curso de Literatura y Medicina para estudiantes de Medicina, que en gran parte se pueden adaptar al MIR de Medicina Interna.

Los textos científicos al uso apenas nos sirven para la patobiografía. En este sentido, los textos literarios tienen una función vicariante muy importante.

- Las repercusiones biográficas de la enfermedad.

\section{TABLA I}

LITERATURA Y MEDICINA. ESBOZO DE UN PROGRAMA DE DOCENCIA COMPLEMENTARIO

1. Clases prácticas alumnos de $3^{\circ}$

2. Pases de visita en sala

3. Sesiones clínicas paralelas

4. Textos literarios

-Repercusiones biográficas de la enfermedad

-Relato en primera persona

-Relación médico-enfermo

-Sociedad y enfermedad

-Ética de la investigación médica 
El pabellón $N^{o} 6$ de Chejov y La muerte de Iván Ilich son obras modelo de la enfermedad como "fractura biográfica." $(41,70)$.

-Relato en primera persona.

Visión de primera mano de las vivencias generadas por la enfermedad (Monte Sinaí, J.L.Sampedro) (71), o el relato de la enfermedad de un hijo (Mortal y rosa, F.Umbral) (72).

- La relación médico-enfermo.

La obra más sugestiva para médicos de familia e internistas es La enfermedad de Sachs, de Martin Winckler (23). Constituye un valioso material docente por las escenas de un sorprendente realismo de la relación clínica.

- Sociedad y enfermedad.

Sin duda, la afección de los enfermos influye en la conducta social. Y la aceptación o marginación del enfermo por parte de la sociedad repercute sobre la vivencia personal de la enfermedad, por ejemplo en infección VIH-SIDA (73), el síndrome de fatiga crónica (74). La autora norteamericana Susan Sontang analiza con detalle estos aspectos en La enfermedad como metáfora (75).

-Ética de la investigación médica.

Las publicaciones del veterinario coreano Woo-Suk Hwang, protagonista de la falsa clonación de células madre humanas, nos hacen reflexionar sobre los límites de la investigación (76). La historia de los grandes científicos (77) proporciona conocimientos y actitudes hacia la ética y el derecho médico.

\section{ADMONICIONES DE EXPERTOS}

Aunque la reticencia irónica de Javier Marías (78) "el atrevimiento y la simplonería de los llamados expertos -en cualquier asunto- resultan deprimentes y a menudo insultantes", puede ser cierta, algunas admoniciones de "expertos" (escritores científicos) sobre la lectura tienen algún valor. Así, Juan Goytisolo invita a los jóvenes a aceptar el desafío de los libros que obligan a la reflexión y a la relectura, y sobre todo a diferenciar entre "texto literario" y "el producto editorial" (79). Navarro, médico hematólogo, opina como Borges: "no hay que leer muchos libros, sino varias veces los mismos libros." (80). Ramón Valls, ex jesuita, advierte que si quieres aprender a pensar, lee librotes y no libritos (81). Wagenberg considera que las personas que no leen ningún libro, tienen más arreglo que las que leen uno solo (59). Desde mi punto de vista, es mejor el escepticismo ante "expertos o guías de lectura", en un campo tan libre, autónomo e individual como es la literatura. Además, estas admoniciones son menos válidas en el mundo científico. Un librito del creador de la mecánica cuántica ondulatoria, Erwin Schrödinger, ¿Qué es la vida? (82), publicado en 1944, tuvo un gran impacto en posteriores concepciones científicas, incluso en Francis Crick (83).

\section{DECÁLOGO DE SUPERSTICIONES}

En la tabla II se detallan las supersticiones, que desde mi experiencia pueden ser perjudiciales en la formación complementaria del MIR en humanidades. Como afirma el
TABLA II

DECÁLOGO DE SUPERSTICIONES

1. Tener que acabar un libro

2. Leer libros llamados importantes. No leer ningún best-seller

3. Leer un solo libro a la vez

4. No escribir en ellos

5. La lectura es un hábito que exige disciplina

6. Tiene mala crítica en el Suplemento Cultural

7. No leer ningún libro publicado en los últimos 50 años

8. No está en el canon de Harold Bloom

9. Bastante tengo con la novela de mi vida

10. Internista y literatura: ¿más "rascador de todas las superficies"?

escritor Alberto Manguel (84): "un verdadero lector no debe creer en estas cosas." El internista como "rascador de todas las superficies" merece otra ironía. Los primates homínidos (Grandes Simios) se transformaron en primates humanos -la humanización- porque crearon instrumentos para rascar superficies, entre otras utilidades (61). Y a propósito del Proyecto del Gran Simio, lo he relacionado con Borges -a más experiencia más áreas relacionables-. Decía Borges: “¿Saben ustedes por qué no hablan los monos? Porque saben que pronto les pondríamos a trabajar." Y, añado yo, quizás "en la Puerta."

\section{LENGUAJE MÉDICO}

Para López Piñero la terminología médica actual es el sedimento de 25 siglos de medicina científica (85). Sin embargo, Pérez Peña (86) y Viana Alonso (87) han hecho especial énfasis en el deterioro del lenguaje médico: en la forma de expresarse, oralmente y por escrito, el imperio de las siglas, la redacción críptica de los informes clínicos, etc., y su grave repercusión en el método clínico y la relación médicoenfermo (86). Terés et al. (28) destacan, entre otras habilidades comunicativas del MIR, la capacidad para redactar, de forma coherente, concisa, legible y comprensible, los documentos clínicos habituales, que, además de uso clínico, pueden serlo también de uso legal, y eviten las siglas, así como juicios de valor sobre el enfermo.

Sin duda, la literatura puede colaborar a la formación médica, a recuperar el lenguaje que permite entendernos y respetar al enfermo. Estos son los fines de la revista Literature and Medicine, que existe desde hace casi 25 años (88).

\section{NUEVOS TIEMPOS}

En mi opinión existen cinco grandes líneas de cambios evolutivos: a) El universo de los blogs (el blognovelista, la blogosfera en donde 25 millones de personas escriben su blog) (89); b) El libro electrónico; c) El papel electrónico; d) Escritores con patrocinio. La novela como nuevo soporte 
publicitario. En España se ha publicado Conexión Bulgari, la novela de Fay Weldon, con el patrocinio de una joyería; y e) Adiós a la literatura terapéutica, a la literatura en torno al "me duele España", "España como problema", "a qué llamamos España" y otros males de la patria.

\section{EPICRISIS}

Es necesario añadir al perfil de médico del siglo XXI las humanidades médicas $(28,90,91)$. Las obras literarias, como afirma Luis Montiel en su magistral conferencia Medicina y Literatura, pueden hacer al lector médico, sin ningún género de dudas, "mejor médico".
No sé si les he persuadido o no, si están a favor o en contra de este discurso, casi prefiero lo último, porque "estar a favor une menos que estar en contra." (92), o como dice Pablo Neruda: "para que nada nos separe que nada nos una."

A lo último, la mejor epicrisis en estas divagaciones es contar los cuentos más cortos y misteriosos de la historia. "Cuando me habló comprendí que el muerto era yo y no él." (Luciano G.Egido, Equívoco) (93). Y el más famoso: “cuando desperté, el dinosaurio todavía estaba allí." (El dinosaurio, Augusto Monterroso) (94).

Queridos Médicos Residentes de Medicina Interna: muchas gracias y "que la incertidumbre os sea favorable." (59).

\section{Bibliografía}

1. Barbado Hernández FJ. Lágrimas perversas. El País, 16-10-2005.

2. Pessoa F. Libro del desasosiego. Ediciones Acantilado. Barcelona, 2002.

3. Brines F. Antología poética. Edit. Espasa. Madrid, 2006.

4. Neruda P. Confieso que he vivido. Memorias. Edit.Seix Barral. Barcelona, 1974.

5. Arribas MA. Bibliófilos versus bibliómanos. Noticias Médicas, 2006; 3879: 58

6. Baroja P. Desde la última vuelta del camino. Edit. Planeta. Bacelona, 1970.

7. Gutiérrez Recadia P. Cuerpo, intimidad, alma. JANO 2006; 1609: 72-4.

8. García-Campayo J, Aseguinolaza L, Tazón P. El desarrollo de las actitudes humanistas en Medicina. Med Clín (Barc) 1998; 111: 23-6.

9. Kasper DL, Fauci AS, Longo DL, Braunwald E, Hauser SL, Jameson JL La práctica de la Medicina. En: Harrison, Principios de Medicina Interna, $16^{\mathrm{a}}$ edic. Barcelona: Mc Graw Hill Edit., 1-6.

10. Baños JE. El valor de la literatura en la formación de los estudiantes de Medicina. http://www.medtrad.org/pana.htm.

11. Marañón G. La Medicina y nuestro tiempo. Espasa-Calpe S.A. Madrid, 1980.

12. Puerta JL. Reflexiones en torno a cinco hitos de la Historia de la Medicina, que ayudan a comprender la práctica médica actual. En: Rodés Teixeidor J, García Massó J. Medicina Interna, $2^{\mathrm{a}}$ ed. Barcelona: Edit.Masson, 2004, p. XLI-XLIX.

13. Ramírez Guerrero M. Los médicos y la literatura. Editor Fundación Unicaja. Málaga, 1986.

14. Médicos escritores. http://www.fisterra.com/human/4miscelanea/ varios/medicos/Escritores.asp

15. Soto Viñolo J. Anton Chéjov murió tuberculoso. JANO, 2004; 1529: 77

16. Gallego V. Chéjov. Ediciones del Orto. Madrid, 1998.

17. Lorenzo-Cáceres Ascanio A. Las humanidades en la formación del médico. Tribuna Docente, 2002; 3: 3-12.

18. Gracia Guillén D. Contribución de las Humanidades Médicas a la formación del médico. Humanitas. Humanidades Médicas. http://www.fundacionmhm.org/tema061/iautor.htlm

19. Pujol Farriols R. Comentario editorial. Humanitas. Humanidades Médicas. http://www.fundacionmhm.org/tema061/iautor.htlm

20. Laín Entralgo P. Medicina e historia. Ediciones Escorial. Madrid, 1941.

21. Laín Entralgo P. La relación médico-enfermo. Alianza Universidad. Madrid, 1983

22. Carreras J, Aitan T. Estudios sobre médicos-filósofos españoles del siglo XIX. Consejo Superior de Investigaciones Científicas. Barcelona, 1952

23. Winckler M. La enfermedad de Sachs. Ediciones Akal. Madrid, 1999.

24. Navarro F. Literatura médica. Diario Médico, 10-5-2006.

25. Camus A. La peste. Editorial Edhasa. Barcelona, 1996.

26. Caballé A Carlos Castilla del Pino. Ediciones Península. Barcelona, 2005.
27. Osler W. Aequanimitas. Fundación Uriach. Barcelona, 2004.

28. Terés J., Capdevila JA, Novell F, Cardellach F, Bundó M, Torres M. Formación troncal de las especialidades médicas: Un reto del presente para una mejora del sistema sanitario. Med Clín (Barc) 2006; 127: 139-44.

29. Millas JJ. Tres miradas. Punto de Lectura S.L. Madrid, 2005.

30. D'Ors Pérez JP. La Medicina Interna como especialidad. Cuadernos de Medicina Interna. Hospital Militar Gómez Ulla. Madrid, 1956.

31. Rof Carballo J. Desarrollo de la patología psicosomática. En: Patología Psicosomática. Edit. Paz Montalvo, 1955, Madrid: 25-44.

32. Laín Entralgo P. La historia clínica. Historia y teoría del relato patobiográfico. Editorial Triacastela. Madrid, 1998.

33. Carrel A. El ser humano y el individuo. En: La incógnita del hombre. Editor Joaquín Gil, 1946. Buenos Aires: 217-47.

34. Laín Entralgo P. Descargo de conciencia. Barral Editores. Barcelona, 1976.

35. Steele SJ, Morton DJ. The ward round. Lancet, 1978; 1: 85

36. Munthe A. La historia de San Michele. Editorial Juventud S.A. Barcelona, 1935 .

37. Aldecoa J, García Montero L, Grandes A, Landero L, Llamazares J, Martín Garzo G, et al. Con otra mirada: Una visión de la enfermedad desde la literatura y el humanismo. Editorial Taurus. Madrid, 2001.

38. Mann T. La montaña mágica. Plaza y Janés Editores S.A. Barcelona, 1995.

39. Cela CJ. Pabellón de reposo. Ediciones Destino S.A. Barcelona, 1989.

40. Landero L. Tumbados y resucitados. Fundación de Ciencias de la Salud. Madrid, 2005

41. Tolstoi L. La muerte de Iván Ilich. Alianza Editorial. Madrid, 2005.

42. Webb LK. Charles Dickens. Ediciones Folio S.A. Barcelona, 1985.

43. Stendhal. Historia de la pintura italiana. Espasa-Calpe S.A. Buenos Aires, 1948

44. Kundera M. La insoportable levedad del ser. Tusquets Editores. Barcelona, 1991

45. Huxley A. Las puertas de la percepción. Cielo e infierno. Editorial Edhasa. Barcelona, 1999.

46. Huxley TH. Introducción al estudio de la ciencia. F. Granada y $\mathrm{C}^{\mathrm{a}}$, editores. Barcelona, 1906.

47. Huxley J. Ensayos de un biólogo. Editorial Sudamericana. Buenos Aires, 1967.

48. Clark RW. Semblanza de sir Julian Huxley. Revista de Occidente 1976; 3: $58-66$.

49. Rodríguez Cuartero A. Charcot, fundador de la neurología: vida y obra. Invest.Clín. 2005; 8: 376-9.

50. Albarracín Teulón A. Freud en París. Medicina e Historia 1966; 18: 1 19.

51. Castilla del Pino C. Pretérito imperfecto. Tusquets Editores. Barcelona, 1997.

52. Baroja P. El árbol de la ciencia. Alianza Editorial. Madrid, 1995.

53. Baroja P. El mundo es ansí. Espasa-Calpe S.A. Madrid, 1964. 
54. Sánchez-Ostiz M. Pío Baroja a escena. Espasa-Calpe S.A. Madrid, 2006.

55. Stendhal. Vida de Henry Brulard. Editorial Alfaguara. Madrid, 2004.

56. Bulgakov M. Morfina. Editorial Anagrama. Barcelona, 2002.

57. Namora F. Escenas de la vida de un médico. Editorial Noguer. Barcelona, 1976.

58. Rozman C. Fundamentos de la práctica médica. En: Farreras-Rozman, Medicina Interna, $15^{\mathrm{a}}$ edic. Madrid: Ed. Elsevia, 2004, p. 3-6.

59. Wagensberg J. Si la naturaleza es la respuesta ¿cuál es la pregunta? Tusquets Editores. Barcelona, 2003.

60. Margulis L. La teoría de la evolución: ¿por qué somos como somos? Fundación Santander Central Hispano. Madrid, 2006.

61. Carbonell E. Somos humanos porque nos hemos hecho complejos. En Los sueños de la evolución. National Geographic, 2003. Barcelona, p. 107-18.

62. Sánchez Ron JM. Diccionario de la ciencia. Editorial Planeta S.A. Barcelona, 1996.

63. Ortega Bargueño P. Jonathan Franzen. El Mundo, 23-4-2002.

64. Steele SJ, Morton SJB. The ward round. Lancet, 1978; 1: 85.

65. Martínez L, de Letona J, Buckley R, Oya M. La enfermedad en la novela de Delibes. En: Enfermedades cardiovasculares, nutrición, genética y epidemiología. Editores Oya M, Garcés C. Ediciones Doyma S.A., 2000. Barcelona, p. 361-77.

66. Baroja P. Miserias de la guerra civil. Editorial Caro-Raggio. Madrid, 2006.

67. Prieto J. El impacto de los sistemas simuladores. JANO, 2003; 1494: 1384.

68. Barbado Hernández FJ, Ramírez J, Sánchez M. Mujer de 50 años con fiebre e infiltrados pulmonares. Med Clín (Barc) 2003; 121: 588-94.

69. Barbado Hernández FJ, Gómez Román J. Conferencias clínico-patológicas de la SEMI. Rev Clín Esp, 2006; 206: 343-52.

70. Gómez Esteban R. El médico como persona en la relación médicopaciente. Editorial Fundamentos. Madrid, 2002.

71. Sampedro JL. Monte Sinaí. Plaza y Janés Editores. Barcelona, 1998.

72. Umbral F. Mortal y rosa. Unidad Editorial S.A. Madrid, 1999.

73. Cooke N, Sande MA. The HIV epidemic and training in internal medicine. N Eng J Med 1989; 321: 1334-8.
74. Barbado Hernández FJ, Gómez Cerezo J, López Rodríguez M, Vázquez Rodríguez JJ. El síndrome de fatiga crónica y su diagnóstico en Medicina Interna. An Med Interna (Madrid) 2006; 23: 238-44.

75. Sontag S. La enfermedad y sus metáforas. Punto de Lectura. Madrid, 2003.

76. Sampedro J. Hwang falsificó sus datos de clonación. El País, 24-122005.

77. Di Trocchio F. Las mentiras de la ciencia ¿por qué y cómo engañan los científicos? Alianza Editorial. Madrid, 1993.

78. Marías J. La intromisión que no para. El País Semanal, 8-1-2006.

79. Aznárez JJ. Goytisolo anima a los jóvenes a releer. El País, 1-12-2004.

80. Andrino. La otra cara de José Luis Navarro. Periódico Informativo Hospital Ramón y Cajal. Madrid, mayo 2006.

81. Lluis Amiguet. Ramón Valls, filósofo. La Vanguardia, 6-12-2003.

82. Schrödinger E. ¿Qué es la vida? Ediciones Orbis S.A. Barcelona, 1986.

83. Sánchez Ron JM. El jardín de Newton. La ciencia a través de su historia. Editorial Crítica. Barcelona, 2001.

84. Luzón J. Leer es la vida. El País Semanal, 18-12-2005.

85. López Piñero JM, Terrada ML. Introducción a la terminología médica. Barcelona. Ed. Masson. 2005

86. Pérez Piña F. Deterioro del lenguaje médico. An Med Interna (Madrid) 1994; 11: 107-8.

87. Viana Alonso A, Morena Fernández J. Abreviaturas o siglas en los informes de alta en Medicina Interna. An Med Interna (Madrid) 1998; 15: 194-6.

88. Rabuzzi KA. Toward a new discipline. Lit Med 1982; 1: 1-118.

89. Estabella A. El universo de los blogs. El País, 19-1-2006.

90. Cardellach F, Vilardell M. Hacia el papel de médico que necesita la comunidad. Med Clín (Barc) 2006; 127: 136-8.

91. Montiel L. Medicina y literatura. http://www.medynet.com/elmedico/ congresos/semergen/abstracts/21-26.pdf

92. Wagensberg J. A más cómo, menos por qué. Tusquets Editores. Barcelona, 2006.

93. Mora M. Luciano G. Egido, escritor. El País, 5-12-2003.

94. Monterroso A. Cuentos, fábulas y lo demás es silencio. Ediciones El País, Clásicos del Siglo XX. Madrid, 2003. 